\title{
Interception of a Moving Object with a Specified Approach Angle by a Wheeled Robot: Theory and Experiment
}

\author{
Ian R. Manchester ${ }^{\dagger}$, Emily-M.P Low*, Andrey V. Savkin*
}

\begin{abstract}
This paper presents a vision-based wheeled-robot navigation technique, termed circular navigation guidance (CNG), for the interception of a moving target from a specific angle. The guidance law is not split into path-planning and path-following stages, but is continuously updated based on immediately available information, making it useful even against a manoeuvring target, or where only incomplete information is available to the robot. This is achieved by converting a finite-time navigation problem into one of maintaining a certain geometric condition at all times. Detailed theoretical analysis provides a closed-form solution for the shape of the path the robot takes against a moving target. Experimental results are presented which show that CNG is robust and accurate under real-world conditions against a manoeuvring target.
\end{abstract}

\section{INTRODUCTION}

In this paper, we present a navigation law by which a wheeled robot can intercept a moving target from a particular approach angle. Such a system has a wide range of potential uses, from intercepting the ball or a player in a game of robot soccer, to a broad spectrum of military and security applications. Despite the potential applications, the problem of approach-angle constrained interception has had comparatively little attention from the robotics community.

We provide detailed theoretical results proving perfect interception and giving a formula for the path the robot takes. The system was implemented on a robotic platform with a video camera as the main sensor, performed successfully in tests against a manoeuvring target.

The navigation law is "reflexive" in nature, i.e. it is based entirely on immediately available information: there is no division into path-planning and path-following stages. This property means it can be easily combined with reflexive systems for other navigation requirements such as obstacle avoidance.

\section{A. Previous Research on Interception}

The key difference between the present navigation strategy those previous considered is the specification of an approach angle. Interception of a moving object, without such a constraint, is a problem well studied from a variety of perspectives, including robotics, game theory, and missile guidance.

$\dagger$ (Corresponding Author) Department of Applied Physics and Electronics, Umeå University, SE-901 87 Umeå, Sweden. Email: Ian.Manchester@tfe.umu.se

* School of Electrical Engineering and Telecommunications, University of New South Wales, UNSW Sydney, 2052, Australia.

This work was supported by the Australian Research Council.
Tracking and grasping of a moving object with a fully actuated industrial robot arm has been studied before, see, e.g., [1], [2], [3]. However, these techniques are hampered by the well-documented nonholonomic constraint on wheeled robots: it is impossible to "slide" sideways, but net motions to the side are possible.

The problem can be posed as a two-player differential game, known as a pursuit-evasion game, as pioneered by Isaacs [4]. Here an intelligent pursuer and evader each use optimal strategies, resulting in a Nash or Stackelberg equilibrium of strategies. However, finding optimal feedback strategies for a realistic nonholonomic robot models and a specified approach angle is not computationally feasible. Linearization about the collision trajectory leads to a soluble problem [5], having many connections with $H^{\infty}$ control theory [6], [7]. In fact, the classical proportional navigation guidance law (see, e.g., [8]) is optimal for this simplified system. However, the problem of interception with a specified approach angle and a nonholonomic wheeled robot is essentially nonlinear, and does not lend itself to such analysis. In contrast, our approach is designed specifically for such nonlinearities, although no claims can be made about optimality.

The authors of [9] propose a method for intercepting a moving object with a mobile robot based on the line-of-sight navigation techniques well known from the missile guidance literature, and the authors of [10] propose and implement a vision-based interception technique. However, these methods do not allow one to specify the angle of approach.

Our control law extends recent results on navigation to a stationary goal [11] and has similarity with recent developments in precision missile guidance with an impact-angle constraint [12]. The present work builds upon this with a much stronger theoretical analysis as well as experimental validation.

\section{PROBLEM FORMULATION}

In this section, we describe the kinematics of the robot, the measurements available to it, and then state the control problem mathematically.

To define the required quantities, it is helpful to consider a Cartesian "world" reference frame with coordinates $x$ and $y$. However, the resulting control law, coming as it does from geometrical principles, is independent of reference frame chosen, and could be calculated in, e.g., the robot's body frame. The variables we now introduce are visualized in Figure 1. 


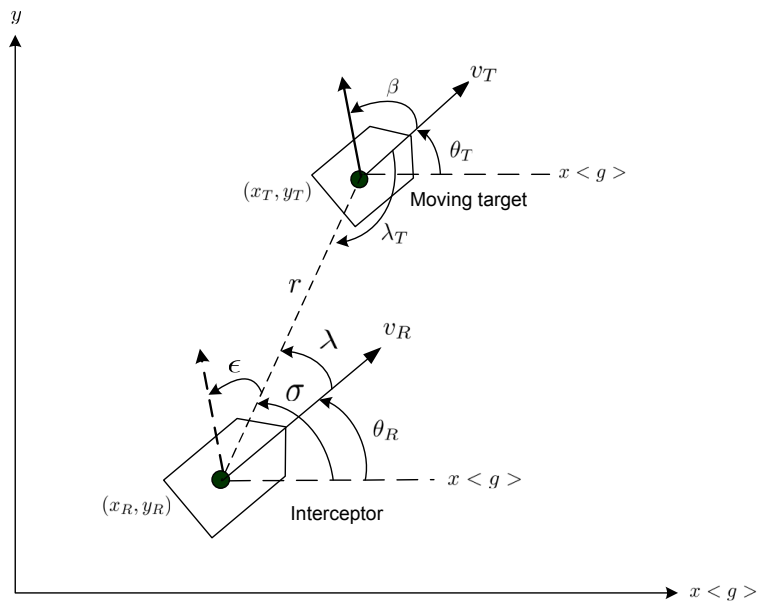

Fig. 1. System geometry of an interceptor and a moving target.

Let $\left(x_{R}, y_{R}\right)$ denote the coordinates of the pursuer robot, and $\left(x_{T}, y_{T}\right)$ the coordinates of the target. Let $\theta_{R}$ and $\theta_{T}$ denote their heading angle, and $v_{R}$ and $v_{T}$ their forward speeds. We consider a simple kinematic model of the robots, often referred to as the "unicycle" model:

$$
\begin{gathered}
\dot{x}_{R}=v_{R} \cos \theta_{R}, \dot{y}_{R}=v_{R} \sin \theta_{R}, \dot{\theta}_{R}=u, \\
\dot{x}_{T}=v_{T} \cos \theta_{T}, \dot{y}_{T}=v_{T} \sin \theta_{T}, \dot{\theta}_{T}=\omega_{T},
\end{gathered}
$$

where $u$ is the control signal we must choose, and $\omega_{T}$ manoeuvre of the target, which not known in advance.

The range between them is $r=\sqrt{\left(x_{T}-x_{R}\right)^{2}+\left(y_{T}-y_{R}\right)^{2}}$. For an intercept to occur, the range must go to zero.

The current "approach angle" is denoted by $\sigma$ and defined as:

$$
\sigma:=\tan ^{-1}\left(\frac{y_{T}-y_{R}}{x_{T}-x_{R}}\right),
$$

where $\tan ^{-1}$ refers to the four-quadrant arc-tangent, which has the whole circle $(-\pi, \pi]$ as its range.

Our aim is to guide the pursuer robot to the target robot with a particular approach angle $\sigma$, relative to the target robots heading. We define the desired approach angle as

$$
\sigma^{*}:=\theta_{T}+\beta
$$

where $\beta$ is chosen by the designer. It would be straightforward to modify our approach to achieve a desired approach angle defined in another way, e.g. independently of $\theta_{R}$.

We now introduce the "approach angle error":

$$
\varepsilon:=\sigma^{*}-\sigma=\theta_{T}+\beta-\sigma .
$$

We would like to drive this quantity to zero, along with the range.

\section{A. Measurements}

Here describe, in terms of the above-defined variables, what measurements are available to the control law. Later in the paper we will describe the actual measurement system implemented in practice.
We define the "line of sight" angles like so:

$$
\begin{aligned}
\lambda & :=\sigma-\theta_{R}, \\
\lambda_{T} & :=-\pi+\sigma-\theta_{T} .
\end{aligned}
$$

These represents the location of the target in the robot's field of view, and the position of the robot in the target's field of view, respectively. We assume that the robot has measurements of $\lambda$, available from the video camera, and can calculate its derivative, $\dot{\lambda}$. The angle $\lambda_{T}$ will be used later in discussions the paper.

We further assume the robot can obtain estimates of $\theta_{T}$ and $v_{T}$. A method to estimate these from video footage using an extended Kalman filter is given later in the paper. We define the pair $\Gamma_{T}=\left(\hat{\theta}_{T}, \hat{v}_{T}\right)$ as representing the robot's current estimate of these.

We assume that the robot has knowledge of its own state, denoted by the 4-tuple $\Gamma_{R}=\left(x_{R}, y_{R}, \theta_{R}, v_{R}\right)$. Indeed, if the robot's body frame is chosen to define the control law, the variables $x_{R}, y_{R}$, and $\theta_{R}$ are trivial, being all zero.

\section{B. Problem statement}

Our complete problem statement is this. To find a guidance law of the form

$$
u=f\left(\lambda, \dot{\lambda}, \beta, \Gamma_{R}, \Gamma_{T}\right)
$$

such that range and angle error at, $r$ and $\varepsilon$, are simultaneously brought as close as possible to zero.

Corresponding to this, we make the following definition:

Definition 1: A target intercept is considered perfect if there exists a finite time $T$ such that:

$$
\begin{aligned}
r(T) & =0, \\
\lim _{t \rightarrow T} \varepsilon(t) & =0 .
\end{aligned}
$$

Note that a limit is used in Definition 1 since if $r(T)=0$, the angle $\sigma(T)$ is undefined and so is $\varepsilon(T)$.

\section{THE NAVIGATION STRATEGY}

The following strategy has been termed the "circular navigation guidance" (CNG) principle: to regulate the robots heading such that

$$
\lambda=\left(\varepsilon+\lambda_{o f f}\right)
$$

where

$$
\lambda_{o f f}:=\sin ^{-1}\left[\left(v_{T} / v_{R}\right) \sin \beta\right] .
$$

For the unicycle model considered, we can propose for example two simple control laws to achieve the CNG principle: A switching controller:

$$
u= \begin{cases}-2 \dot{\lambda} & \text { if } \lambda-\left(\varepsilon+\lambda_{o f f}\right)=0, \\ u_{\max } & \text { if } \lambda-\left(\varepsilon+\lambda_{o f f}\right)<0, \\ -u_{\max } & \text { if } \lambda-\left(\varepsilon+\lambda_{o f f}\right)>0,\end{cases}
$$

or a proportional + feedforward controller:

$$
u=-2 \dot{\lambda}-k_{p}\left[\lambda-\left(\varepsilon+\lambda_{o f f}\right)\right]
$$

where $k_{p}$ is a positive gain. 


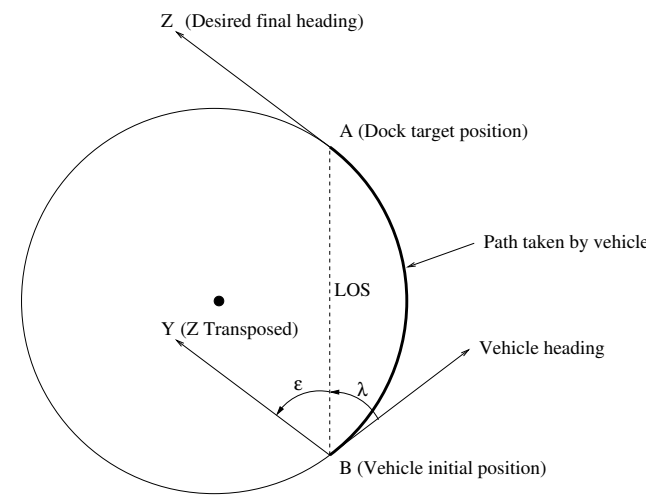

Fig. 2. Geometry for CNG with a stationary target.

\section{A. Discussion of the navigation strategy}

It was shown in [12] that with a stationary target (implying $\lambda_{\text {off }}=0$ ), the condition $\lambda=\varepsilon$ results the guided vehicle taking a circular path to the target - hence the name "circular navigation guidance" - and reaching it with exactly the correct approach angle. That is, $r$ and $\varepsilon$ go to zero simultaneously and in finite time. This is visualized in Figure 2. In the same paper, it was shown that introducing the offset angle $\lambda_{\text {off }}$ results in a perfect intercept of a moving object.

If the intercept target is moving with constant heading (i.e., $\dot{\theta}_{T}=0$ ) then the following dynamical equations are apparent from the definitions (6) and (5):

$$
\begin{aligned}
\dot{\lambda} & =\dot{\sigma}-u, \\
\dot{\varepsilon} & =-\dot{\sigma}, \\
\dot{\lambda}-\dot{\varepsilon} & =2 \dot{\sigma}-u, \\
& =2 \dot{\lambda}+u .
\end{aligned}
$$

Note that $\lambda_{o f f}$ is a constant, so we have also

$$
\frac{d}{d t}\left[\lambda-\left(\varepsilon+\lambda_{\text {off }}\right)\right]=2 \dot{\lambda}+u,
$$

describing the rate of change of the "distance" to satisfaction of the CNG condition.

From this equation, it is clear that the control laws (13) and (14) serve to regulate the CNG condition. The switching control law (13) will clearly drive $\lambda$ to $\varepsilon+\lambda_{\text {off }}$ in finite time as long as $u_{\max }>2|\dot{\lambda}|$.

$$
\dot{\lambda}=\frac{v_{R}}{r} \sin \lambda+\frac{v_{T}}{r} \sin \lambda_{T}-u
$$

hence $u_{\max }>2\left(v_{R}+v_{T}\right) / r$ is a sufficient condition for reduction of error, and the CNG condition will clearly be satisfied in finite time if the initial range is sufficient.

In practice, a smooth control law such as (14) may be preferable to a switching control law, since factors such as unmodeled actuator dynamics and time delays can lead to oscillations and chattering in switching controllers. The proportional control law (14) results in the following error dynamics:

$$
\frac{d}{d t}\left[\lambda-\left(\varepsilon+\lambda_{o f f}\right)\right]=-k_{p}\left[\lambda-\left(\varepsilon+\lambda_{o f f}\right)\right],
$$

Clearly the error $\lambda-\left(\varepsilon+\lambda_{o f f}\right)$ will be a segment of an exponentially convergent signal. Furthermore, it has been shown that such a controller will still result in an intercept with $r$ going to zero in finite time, and the final error in $\varepsilon$ can be made arbitrarily small by increasing $k_{p}$ [12].

For most kinematic and dynamic models of robots and vehicles regulating the heading to a particular value is a wellstudied problem and is quite straightforward.

\section{MAin TheORETICAL RESUlts}

In this section we prove some theoretical results about the navigation law described above.

We define the following values, which are constant for a particular engagement, i.e. initial conditions, robot and target speed, and choice of $\beta$ :

$$
\begin{gathered}
\alpha:=\frac{v_{R} \cos \lambda_{o f f}-v_{T} \cos \beta}{v_{R} \cos \lambda_{o f f}+v_{T} \cos \beta}, \\
\gamma:=\frac{v_{T} \sin \beta}{v_{R} \cos \lambda_{o f f}+v_{T} \cos \beta}, \\
\kappa:=\frac{\left(v_{R} \cos \lambda_{o f f}+v_{T} \cos \beta\right)|\sin \varepsilon(0)|^{\alpha}}{r(0) e^{\gamma \varepsilon(0)}} .
\end{gathered}
$$

It will be shown that $\alpha, \kappa>0$ and that, in a certain sense, $\alpha$ characterizes the "difficulty" of the intercept.

Theorem 1: If the CNG condition $\lambda=\varepsilon+\lambda_{\text {off }}$ is satisfied, then

1) If $\varepsilon(0)=0$ then the robot takes a straight line path and reaches the target in finite time with the correct approach angle.

2) If $\varepsilon(0)=\pi$ then the robot takes a straight line path away from the target and the intercept fails.

3) If $\varepsilon(0) \neq 0$ or $\pi$ then the robot reaches the target in finite time with the correct approach angle, the shape of its path in $(r, \varepsilon)$ space is given by the algebraic equation:

$$
r(t)=r(0)\left(\frac{\sin \varepsilon(t)}{\sin \varepsilon(0)}\right)^{\alpha} e^{\gamma(\varepsilon(t)-\varepsilon(0))},
$$

and the approach angle error $\varepsilon$ are obeys the following first-order differential equation:

$$
\dot{\varepsilon}=-\kappa \operatorname{sign}(\varepsilon)|\sin \varepsilon|^{1-\alpha} e^{\gamma \varepsilon} .
$$

Proof omitted to meet space restrictions.

We now discuss some qualitative aspects of the path described by (17) and (18).

Note that when the CNG condition is satisfied $u=-2 \dot{\varepsilon}$, and therefore we have also an expression for the required turn rate:

$$
u=2 \kappa \operatorname{sign}(\varepsilon)|\sin \varepsilon|^{1-\alpha} e^{\gamma \varepsilon} .
$$

The theorem states that in all cases except $\varepsilon(0)=\pi$ the intercept is perfect. However, it may happen that as the robot approaches the target, the control effort called for grows 



Fig. 3. $d \varepsilon / d t$ as a function of $\varepsilon$ for different values of the "difficulty factor" $\alpha$.

without bound. Any real mobile robot has a limited turn radius, so this is clearly a limitation for practice.

The term $\alpha$, which can be any positive real number, has a critical effect on the shape of the path, which we now summarize:

- If $\alpha<1$, then the required turn rate goes to zero at the time of intercept,

- If $\alpha=1$, the required turn rate is bounded, but remains non-zero at the time of intercept,

- If $\alpha>1$, the required turn rate grows without bound as the time of intercept approaches.

Figure 3 depicts three example profiles for the differential equation (19) for three different values of $\alpha$, and in Figure 4 a the approach path shapes, calculated from (17), are shown for five different values of $\alpha$. Note that when $\alpha$ is small, the robot approaches the target smoothly from the correct approach angle, whereas with large $\alpha$ it approaches from the side and a sharp correction must be performed in the final moments.

If we examine the structure of the formula for $\alpha$, we see that it is essentially dependant on the target speed, $v_{T}$, and the desired approach angle, $\beta$. The following are clear:

- If $v_{T}=0$, then $\alpha=1$, and close to one if $v_{T}$ is close to zero.

- for any given $v_{T}>0$ :

- $\alpha<1$ if $|\beta|<\pi / 2$ with a minimum at $\beta=0$ (tailon approach);

- $\alpha=1$ for $\beta= \pm \pi / 2$;

- $\alpha>1$ if $|\beta|>\pi / 2$ with a maximum at $\beta=0$ (headon approach).

It is clear from this that the "easiest" intercepts for CNG, in terms of required turn-rate, are tail-on approaches to fast targets, followed by side on approaches or intercepts of a stationary target, and the most difficult are head on



Fig. 4. Shape of the approach, in relative position, for different values of the "difficulty factor" $\alpha$.

approaches of a fast target.

The constants $\gamma, \kappa$ do not have such an effect on the qualitative properties of the path taken, with $\gamma$ providing a "warping" of the paths in Figure 4 due to target motion, and $\kappa$ changing the rate of convergence. However, we note that if $v_{T}=0$ then $\alpha=1$ and $\gamma=0$, hence

$$
r(t)=r(0)\left(\frac{\sin \varepsilon(t)}{\sin \varepsilon(0)}\right),
$$

and

$$
\dot{\varepsilon}=-\kappa \operatorname{sign}(\varepsilon)
$$

which clearly describes the circular path described in the previous section.

\section{A. Performance with inaccurate measurement of target mo- tion}

Using a pan-controlled video camera, it is quite straightforward to obtain a good measurement of $\lambda$. However, good estimates of $\theta_{T}$ and $v_{T}$ may be much more difficult to obtain. In this subsection we show that, even if the robot has erroneous estimates of these values, the miss distance will still be zero, and the angle error will be small, depending on the degree of the errors.

Theorem 2: Suppose a control law regulating the $\mathrm{CNG}$ condition is implemented with incorrect estimates of $\theta_{T}$ and $v_{T}$, denoted $\hat{\theta}_{T}$ and $\hat{v}_{T}$. The robot will be guided to the target at a finite time $T$ with $r(T)=0$. The angle error is continuous with respect to the estimation errors, and therefore will be small if the errors are small.

Proof: The control law with incorrect estimates $\hat{\theta}_{T}, \hat{v}_{T}$ is identical to the control law with true values of $\theta_{T}, v_{T}$, but 
a differently defined desired approach angle $\bar{\beta}$, which is the solution of the following equation:

$$
\bar{\beta}+\sin ^{-1}\left(\frac{v_{T}}{v_{R}} \sin \bar{\beta}\right)=\beta+\left(\hat{\theta}_{T}-\theta_{T}\right)+\sin ^{-1}\left(\frac{\hat{v}_{T}}{v_{R}} \sin \beta\right) .
$$

Therefore, by Theorem $1, r(T)=0$, and $\sigma$ will converge to $\theta_{T}+\bar{\beta}$, and the angle error will be

$$
\lim _{t \rightarrow T} \varepsilon(t)=\beta-\bar{\beta},
$$

which is continuous with respect to $\hat{\theta}_{T}$ and $\hat{v}_{T}$.

The new impact angle $\bar{\beta}$ is implicitly defined by Equation (22). An exact expression for $\bar{\beta}$ can be calculated with computer algebra packages, however the resulting expression is several pages long. Despite this, the sensitivity of $\hat{\beta}$ with respect to the measurement errors can be expressed quite simply:

$$
\begin{aligned}
&\left.\frac{\partial \bar{\beta}}{\partial \hat{\theta}}\right|_{\hat{\theta}=\theta, \hat{v}_{T}=v_{T}}=\frac{\sqrt{v_{R}^{2}-v_{T}^{2} \sin ^{2} \hat{\beta}}}{\sqrt{v_{R}^{2}-v_{T}^{2} \sin ^{2} \hat{\beta}}+v_{T} \cos \hat{\beta}}, \\
&\left.\frac{\partial \bar{\beta}}{\partial \hat{v}_{T}}\right|_{\hat{\theta}=\theta, \hat{v}_{T}=v_{T}}=\frac{\sin \hat{\beta}}{\sqrt{v_{R}^{2}-v_{T}^{2} \sin ^{2} \hat{\beta}}+v_{T} \cos \hat{\beta}} .
\end{aligned}
$$

In particular, one can see that if $\beta=0$ or $\pi$, errors in estimates of the target's linear velocity do not affect the performance of the guidance law. Another conclusion one can draw is that if the pursuer robot is much faster than the target, then errors in target-heading estimate give rise to approximately equal errors in the approach angle $(\partial \bar{\beta} / \partial \hat{\theta} \approx$ $1)$.

\section{EXPERIMENTAL RESUlTS}

In this section we present results from experiments on a real robot platform. Due to space restrictions, we cannot describe the experimental setup in full detail, however it is essentially the same as the setup detailed in [11] with a second Pioneer robot as the target. The pursuer robot uses a video camera as its primary sensor, along with optical wheel encoders to measure its own motion. Such sensors are commonplace and inexpensive, yet provide sufficient information for our control system to achieve its goal. The experimental platform consists of two Pioneer wheeled robots and a pan video camera. An extended Kalman filter is used to estimate the moving target's velocity from visual and odometry measurements.

Our theoretical results on perfect performance were only proved for a constant-velocity target. However, the fact that our guidance law is reactive, i.e. responds to immediately available information, and does not break the task into pathplanning and path-following steps, suggests it may also perform well against manoeuvring targets.

The trajectories taken by the pursuer and quickly manoeuvring target, with a desired approach angle of $\beta=$ $\pi / 2$ radians (i.e. $\alpha=1$ ). are shown in Figure 5 and the

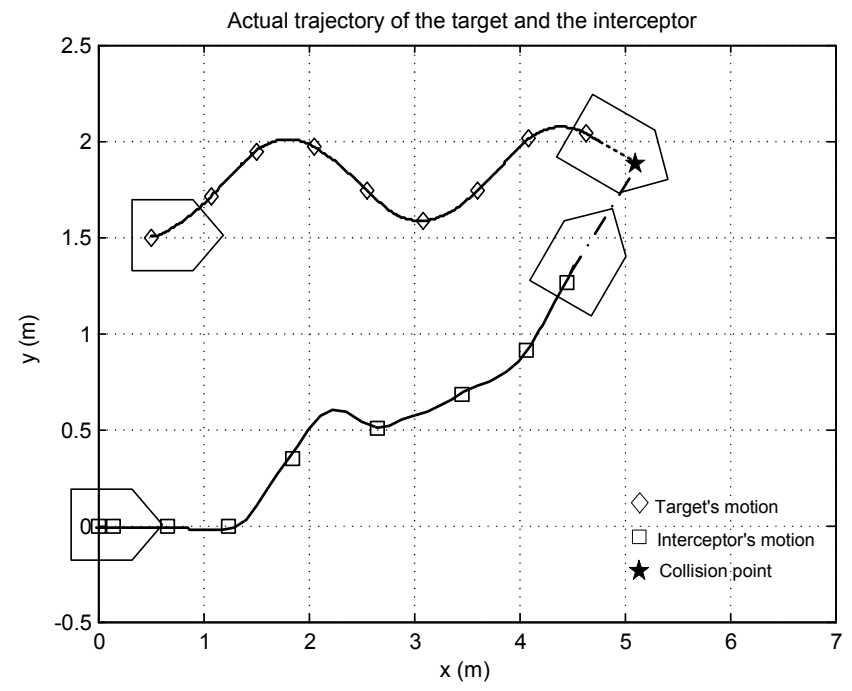

(a) Trajectory of the interceptor intercepting the target.
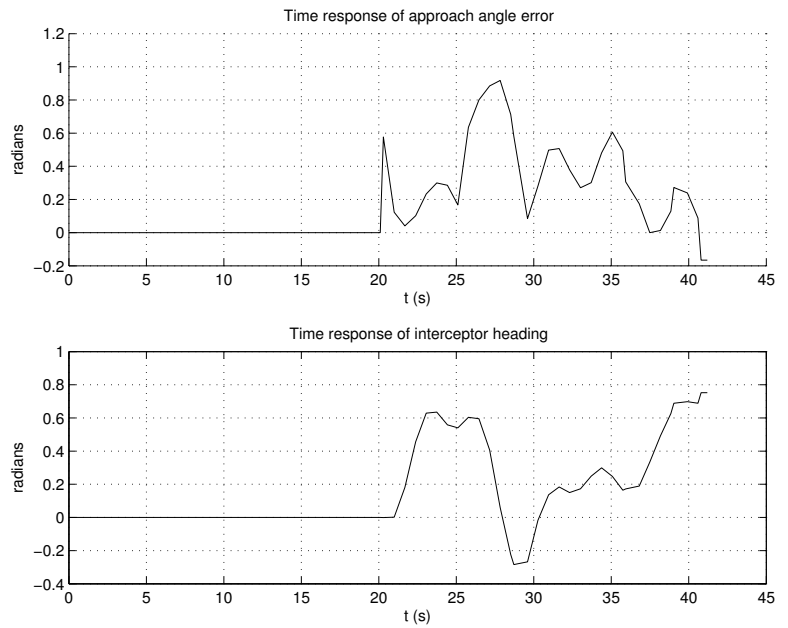

(b) Time response of $\varepsilon$ and $\theta_{R}$.

Fig. 5. Trajectory taken by the interceptor and the highly manoeuvring target $v_{T}=0.1 \mathrm{~m} / \mathrm{s}$ and $\omega_{T}=0.125 \sin \left(0.25 t+\frac{\pi}{2}\right)$ for $\beta=\pi / 2$.

performance of the state estimator is shown in $6 \mathrm{~A}$ sequence of photographs showing this interception take place are given in Figure 7. In this case, it proved difficult to estimate the target's linear velocity, and we can see that the line-of-sight angle $\lambda$ generated from the vision system had quite large errors.

It is important to note that the pursuer still hit the target, with small errors in the approach angle. As such, these experimental results strongly support the theoretical results in Section IV-A and are indicative of the robustness of the CNG principle..

\section{CONCLUSION}

In this paper we have presented a robot navigation technique, circular navigation guidance, which solves the problem of intercepting a moving target from a specific angle, using only data from a video camera. The guidance law 


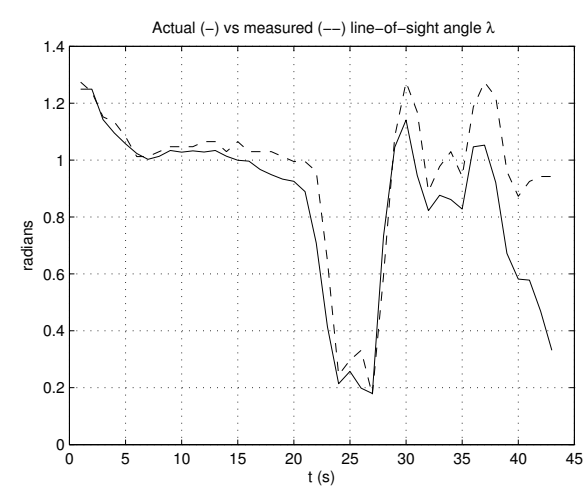

(a) Accuracy of the line-of-sight angle measurements from the vision system.
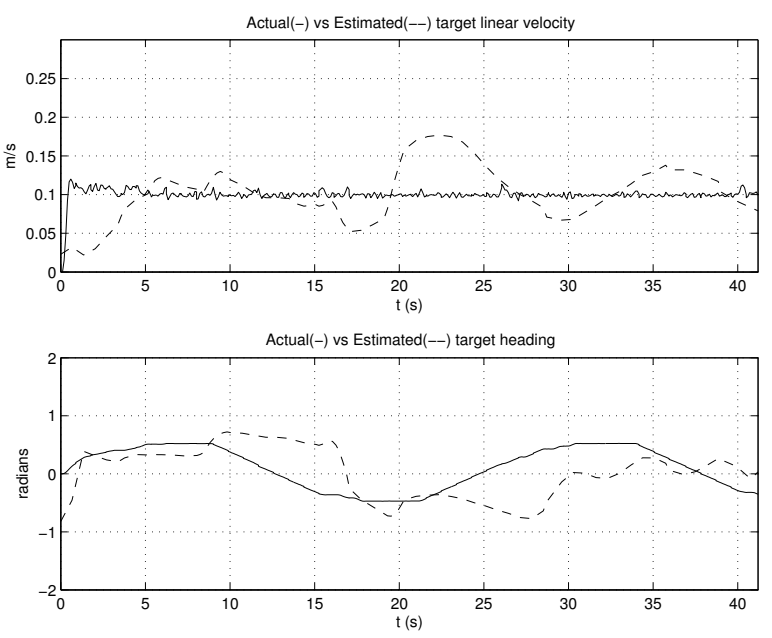

(b) Accuracy of the target's linear velocity and heading estimated by the interceptor based on measurements from the vision system.

Fig. 6. Position and velocity estimation and the highly manoeuvring target $v_{T}=0.1 \mathrm{~m} / \mathrm{s}$ and $\omega_{T}=0.125 \sin \left(0.25 t+\frac{\pi}{2}\right)$ for $\beta=\pi / 2$.

is not split into path-planning and path-following stages, but is continuously updated based on immediately available information, making it useful even against a manoeuvring target, or where only incomplete information is available to the robot. This is achieved by converting a finite-time navigation problem into one of maintaining a certain geometric condition at all times.

Theoretical results were given which prove that the intercept will be perfect under idealized conditions, and give an analytical formula for the path it takes. Furthermore, it was proved that in the practically-important case where the lineof-sight angle of the target is available, but the motion of the target is difficult to estimate, as is the case with a visionbased system, the navigation law will still result in zero miss distance, and only the approach angle will be affected.

Experimental results were presented which show good performance. The success in experiments also prove that the guidance law can be successfully implemented in real-time, and is robust to the numerous measurement and actuation errors which are practically unavoidable on a mobile robotic platform.
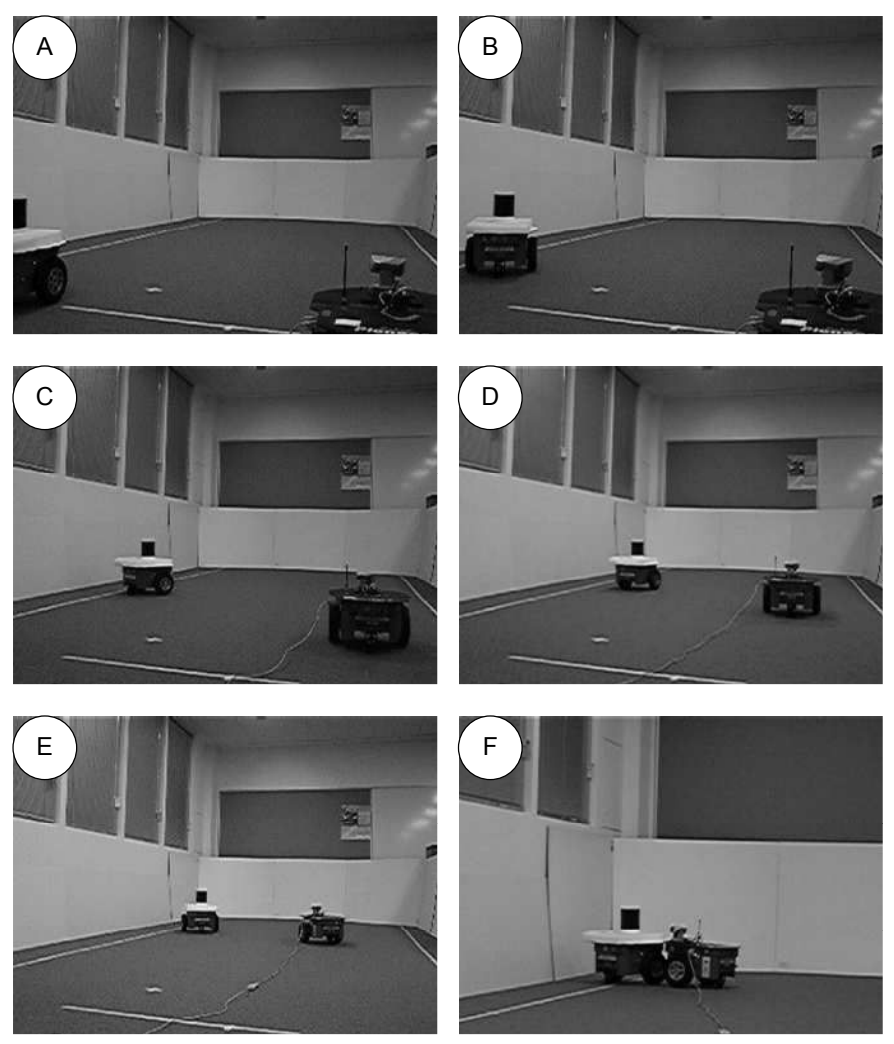

Fig. 7. Photographs of the interceptor and the highly manoeuvring target.

\section{REFERENCES}

[1] P. K. Allen, A. Timcenko, B. Yoshimi, and P. Michelman, "Automated tracking and grasping of a moving object with a robotic hand-eye system," IEEE Transactions on Robotics and Automation, vol. 9, no. 2, pp. 152-165, Apr 1993.

[2] M. Mehrandezh, N. Sela, R. Fenton, and B. Benhabib, "Robotic interception of moving objects using an augmented ideal proportional navigation technique," IEEE Transactions on Systems, Man and Cybernetics, Part A, vol. 30, no. 3, pp. 238-250, May 2000.

[3] D. Hujic, E. Croft, G. Zak, R. Fenton, J. Mills, and B. Benhabib, "The robotic interception of moving objects in industrial settings: strategy development and experiment," IEEE/ASME Transactions on Mechatronics, vol. 3, no. 3, pp. 225-239, Sep 1998.

[4] R. Isaacs, Differential Games. New York: Wiley, 1965.

[5] Y. C. Ho, A. E. Bryson, and S. Baron, "Differential games and optimal pursuit-evasion strategies," IEEE Transactions on Automatic Control, vol. 10 , no. 4, pp. 385-389, Oct 1965.

[6] T. Basar and G. J. Olsder, Dynamic noncooperative game theory, ser. Classics in Applied Mathematics. Philadelphia: SIAM, 1999.

[7] I. R. Petersen, V. A. Ugrinovskii, and A. V. Savkin, Robust Control Design using $H^{\infty}$ Methods. London: Springer-Verlag, 2000.

[8] N. A. Shneydor, Missile Guidance and Pursuit: Kinematics, Dynamics and Control. Chichester, England: Horwood Publishing, 1998.

[9] F. Belkhouche, B. Belkhouche, and P. Rastgoufard, "Line of sight robot navigation toward a moving goal," IEEE Transactions on Systems, Man and Cybernetics, Part B, vol. 36, no. 2, pp. 255-267, April 2006.

[10] F. Capparella, L. Freda, M. Malagnino, and G. Oriolo, "Visual servoing of a wheeled mobile robot for intercepting a moving object," in IEEE/RSJ Conference on Intelligent Robots and Systems, Aug 2005.

[11] E. M. P. Low, I. R. Manchester, and A. V. Savkin, "A biologically inspired method for vision-based docking of wheeled mobile robots," Robotics and Autonomous Systems, vol. 55, no. 10, pp. 769-784, Oct 2007.

[12] I. R. Manchester and A. V. Savkin, "Circular navigation guidance law for precision missile target engagements," Journal of Guidance, Control, and Dynamics, vol. 29, no. 2, pp. 314-320, Mar-Apr 2006. 